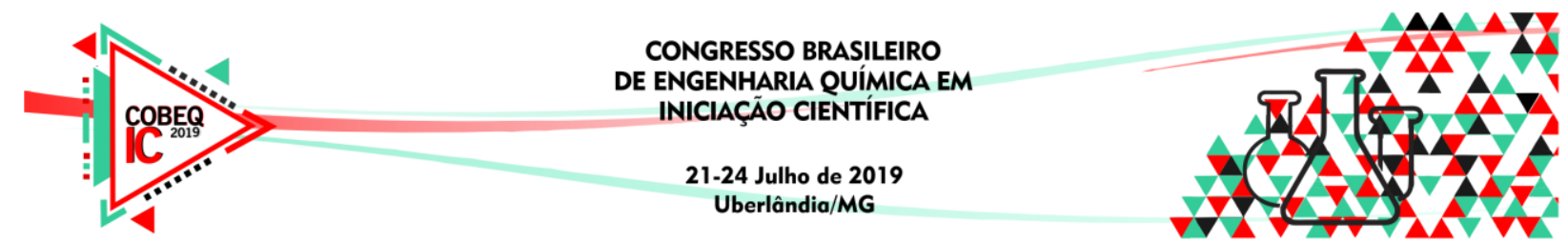

\title{
AVALIAÇÃO EXPERIMENTAL E MODELAGEM DA FILTRAÇÃO UTILIZANDO FLUIDOS DE PERFURAÇÃO NÃO-NEWTONIANOS
}

\author{
L.D.R.ANDRADE ${ }^{1}$, R.F.O.BORGES ${ }^{2}$, B.F.OESCHLER ${ }^{3}$, L.A.CALÇADA ${ }^{4}$, C.M.SCHEID ${ }^{5}$ \\ ${ }^{1}$ Universidade Federal Rural do Rio de Janeiro, Departamento de Engenharia Química \\ ${ }^{2}$ Universidade Federal do Rio de Janeiro, Departamento de Engenharia Química \\ E-mail para contato: lucasrissi01@gmail.com
}

\begin{abstract}
RESUMO - A existência de um gradiente de pressão entre a região anular e o reservatório resulta, durante a perfuração de um poço de petróleo, na perda de fluido para a formação. Quando o volume de fluido perdido é muito grande temos a chamada perda de circulação. Neste processo a rocha reservatória atua como meio filtrante e retém as partículas em suspensão no fluido de perfuração, levando a formação de uma fina camada de reboco que reduz a taxa de invasão dos mesmos. Desta forma, a invasão dos fluidos se torna função das propriedades da torta formada, como porosidade e permeabilidade. Neste cenário, o monitoramento e a capacidade de predição destas propriedades se mostram de fundamental importância. Neste estudo é avaliado o efeito da pressão sobre as propriedades da torta e parâmetros como resistência do meio filtrante, permeabilidade e porosidade de torta. Os estudos foram conduzidos em condição de filtração estática sendo utilizados fluidos de perfuração a base água viscosificados com CMC e adensados com barita. Os resultados demonstram a tendência de redução da porosidade média da torta com o aumento da pressão, assim como o aumento da espessura da torta. A permeabilidade da torta apresenta valor máximo para os níveis de menor pressão seguida por uma redução no parâmetro quando combinado com o aumento da mesma.
\end{abstract}

\section{INTRODUÇÃO}

Na técnica de perfuração overbalance, a pressão na região anular do poço que está sendo perfurado é mantida superior à pressão da formação rochosa. Esta técnica proporciona maior estabilidade operacional, ao passo que necessita de maior controle das propriedades do fluido de perfuração para manutenção da pressão hidrostática e ainda leva a invasão dos fluidos de perfuração para os reservatórios devido ao gradiente de pressão existente (McCABE et al. 1993; NYGAARD \& NÆDVAL, 2006; DA SILVA, 2017).

Neste cenário, a formação rochosa atua como meio filtrante e retém as partículas dos sólidos em suspensão presentes nos fluidos de perfuração. Estas partículas agregadas formam uma camada de reboco sobre a superfície rochosa do poço que reduz a taxa de infiltração dos fluidos de perfuração (CALÇADA et al., 2011).

O processo de filtração que ocorre durante a perfuração vem sendo estudado há anos e diversos trabalhos e modelos vem sendo desenvolvidos, objetivando-se melhor descrever a dinâmica do processo. A teoria simplificada da filtração, desenvolvida por Ruth (1931) considerando fluido newtoniano, tem sido a mais utilizada ao longo de décadas. Da mesma 


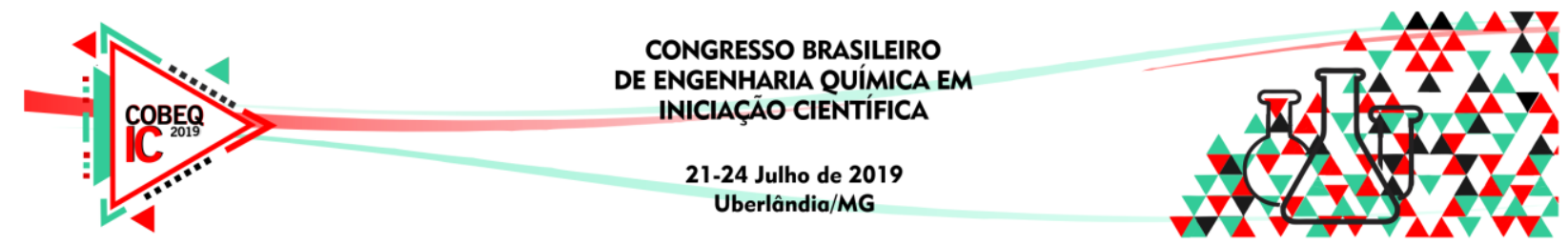

forma, Castro e Massarani (1981) desenvolveram uma extensão a primeira, considerando fluido não-newtoniano. Outras abordagens empíricas e fenomenológicas surgiram com o passar dos anos como os modelos de Dewan e Chenevert (2001), apresentando o conceito da curva de Slowness, e Bürguer et al. (2001) que introduz a teoria da ocorrência da sedimentação em conjunto com a filtração. Estas propostas apresentam eficácia e precisão, derivando de uma estrutura complexa de rotinas de cálculo, demandando cada vez mais dados de campo.

Trabalhos anteriores do grupo procuraram avaliar o efeito da pressão, granulometria, diferentes agentes adensantes e viscosificantes nas propriedades das tortas de filtração (FERRAZ, 2014; DA SILVA, 2017). Da Silva (2017) utilizou a abordagem de Castro e Massarani para ajuste dos dados experimentais, enquanto que os demais trabalhos se valeram da modelagem proposta por Dewan e Chenevert.

Este trabalho tem como objetivo avaliar a dinâmica do processo de filtração estática com formação de torta, a partir de dados experimentais de filtração utilizando fluidos de perfuração base água não-Newtonianos utilizando diferentes faixas de pressão de operação. Os dados foram ajustados ao modelo de Castro e Massarani (1981) para fluidos não newtonianos, para estimar as permeabilidades e compressibilidade das tortas de filtração.

\section{MATERIAIS E MÉTODOS}

\subsection{Preparação dos fluidos e planejamento experimental}

A solução não-Newtoniana foi obtida a partir da preparação de uma solução aquosa de carboximetilcelulose (CMC) na concentração de 5,70 g/L (concentração típica para fluidos de perfuração) utilizando um agitador mecânico Fisatom com agitação constante de 1600 rpm durante 10 minutos. Para o preparo das suspensões, adicionou-se barita à solução de CMC até atingir a concentração de $30 \% \mathrm{v} / \mathrm{v}$ (comum para fluidos de perfuração), mantendo-se a agitação constante em $1600 \mathrm{rpm}$ por 20 minutos.

A barita utilizada possui faixa de distribuição granulométrica entre 0,3 e $60 \mu \mathrm{m}\left(\mathrm{D}_{3,2}=\right.$ $4,54 \mu \mathrm{m})$ e massa específica $3811,6 \pm 0,8 \mathrm{~kg} / \mathrm{m}^{3}$, obtida via técnica de picnometria a gás hélio em quintuplicata.

O fluido base (solução aquosa de CMC) apresenta comportamento não-Newtoniano pseudoplástico. Para os ensaios de reologia, utilizou-se o reômetro HAAKE RheoStress 1 (ThermoFisher Scientific) com a geometria de cilindros concêntricos. Os dados experimentais obtidos nos ensaios reológicos são ilustrados na Figura 1, a qual contempla o ajuste dos mesmos ao modelo do tipo Power-law e a curva de viscosidade aparente $\left(\eta_{\mathrm{ap}}\right)$ em função da taxa de cisalhamento $\left(\gamma^{*}\right)$. Uma triplicata foi realizada de forma a caracterizar o erro experimental. Para o fluido em questão, o índice de consistência estimado foi de 1,148 $\pm 0,03$ Pa. $s^{\mathrm{n}}$ e 0,483 $\pm 0,02$ para o índice de comportamento, a $298 \mathrm{~K}$.

As filtrações foram realizadas utilizando pressões de operação de 2,0 $\mathrm{MPa}, 3,4 \mathrm{MPa}$ e 4,8 MPa. Foi realizada uma triplicata no ponto central experimental $(3,4 \mathrm{MPa})$, de forma a garantir a existência de graus de liberdade suficientes para a realização do tratamento estatístico dos dados. O erro experimental foi caracterizado considerando um nível de confiança de $95 \%$. 


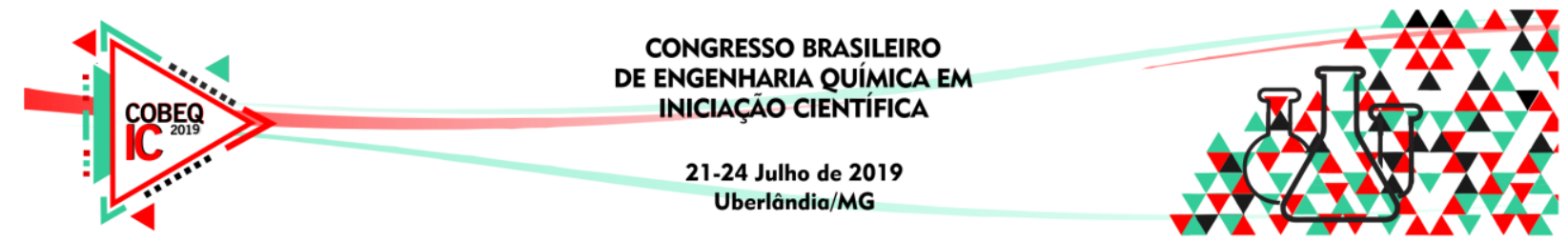

Figura 1 - Dados reológicos experimentais para a solução aquosa de CMC. Curva de tensão e ajuste do modelo Power-law (1.A); e curva de viscosidade aparente (1.B).
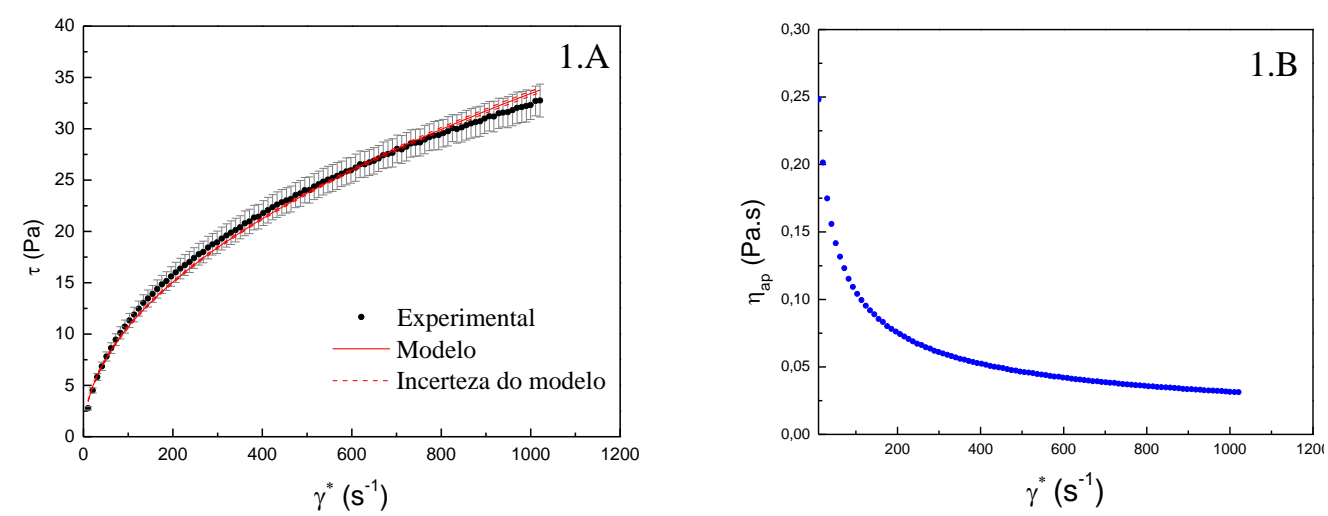

\subsection{Ensaios de filtração}

Foi avaliado o efeito da pressão sobre o volume de filtrado, porosidade, espessura de torta e parâmetros da filtração. A Figura 2 apresenta a célula de filtração HTHP (High Temperature High Pressure) da OFITE® utilizada para os ensaios de filtração. O aparato experimental consiste em uma célula de filtração em aço INOX de aproximadamente 4,9.10 $\mathrm{m}^{3}$, utilizando $\mathrm{N}_{2(\mathrm{~g})}$ como fonte de pressurização (máximo de $14 \mathrm{MPa}$ ). Como meio filtrante foi utilizado papel de filtro FANN $\operatorname{com} 2,3 \cdot 10^{-3} \mathrm{~m}^{2}$ de área útil e retenção de partícula na faixa de 2,7 a $5,0 \mu \mathrm{m}$.

Figura 2: Unidade experimental de filtração HTHP.

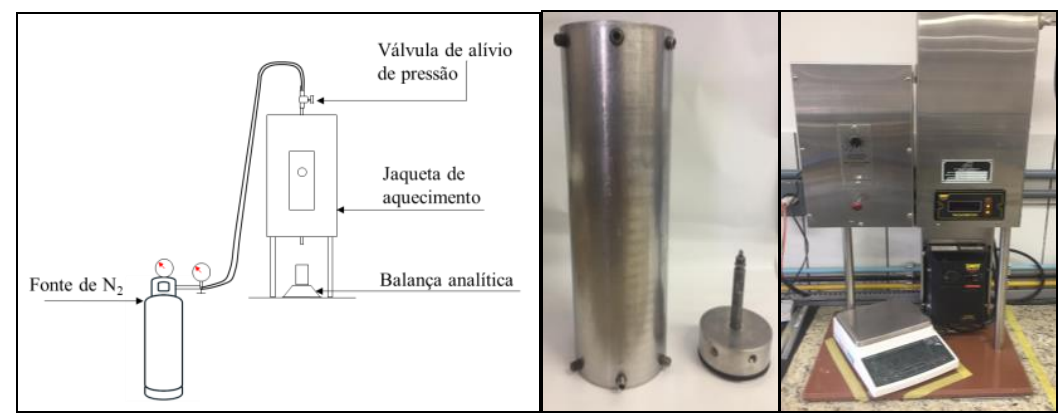

Ao longo dos experimentos foram registrados os dados de volume de filtrado e tempo decorrido, ao longo de 1800s. Ao final do tempo considerado, a torta de filtração foi recolhida para gravimetria e determinação de espessura e porosidade. A espessura das tortas foi obtida utilizando um paquímetro. A porosidade foi calculada a partir da relação massa de torta seca massa de torta úmida, via balanço de massa.

\subsection{Modelagem e estimação de parâmetros}

Para ajuste dos dados experimentais utilizou-se a teoria simplificada da filtração para fluidos não-Newtonianos desenvolvida por Castro e Massarani (1981), agrupando o efeito do parâmetro reológico e do diferencial de pressão nos parâmetros a e b a serem estimados (Equação 1). 


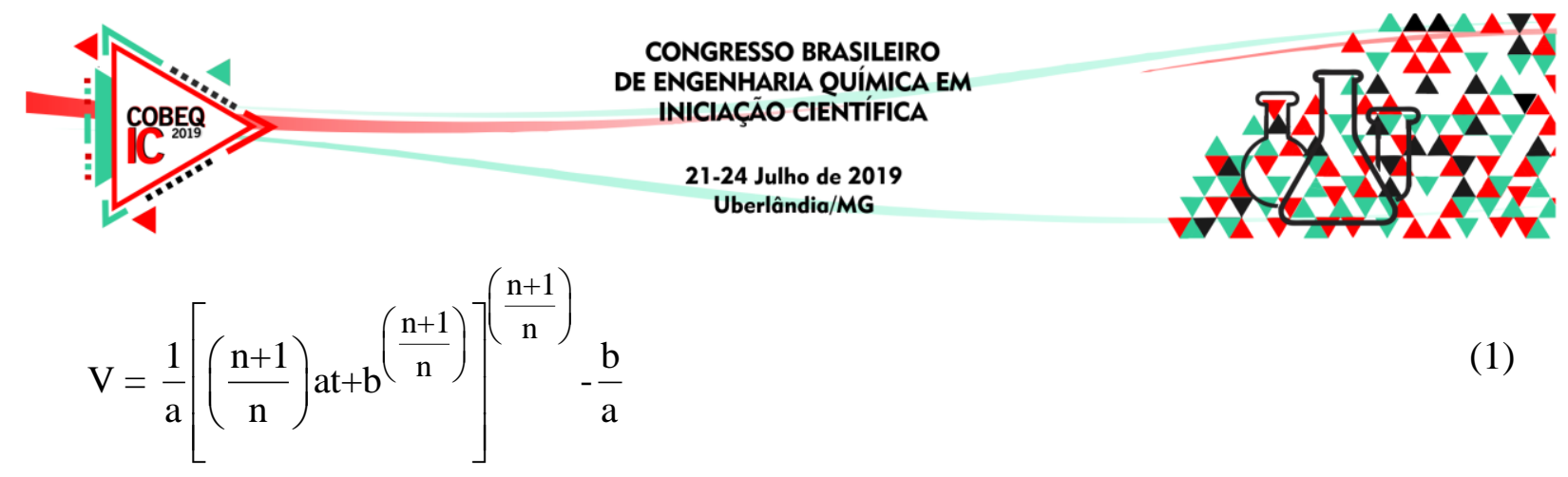

Sendo a e b os parâmetros de ajuste estimados para o modelo. Adicionalmente, V é o volume de filtrado $\left(\mathrm{m}^{3}\right)$, t é o tempo de filtração (s) e $\mathrm{n}$ é o índice de comportamento do fluido nãoNewtoniano segundo o modelo de lei de potência. Deve-se ressaltar que os parâmetros reológicos utilizados são aqueles obtidos para o fluido base (solução aquosa de CMC), sem a presença do sólido adensante.

Os parâmetros a e b estão definidos nas Equações 2 e 3.

$$
\begin{aligned}
& \mathrm{a}=\frac{\mathrm{MC}}{\Delta \mathrm{P}} \frac{\rho_{\mathrm{f}}}{\mathrm{A}^{\mathrm{n}+1}} \frac{1}{\mathrm{k}^{(\mathrm{n}+1) / 2}} \frac{1}{\left(\rho_{\mathrm{s}}\left\langle\varepsilon_{\mathrm{s}}\right\rangle\right)^{1 / 2}} \\
& \mathrm{~b}=\frac{\mathrm{M}}{\mathrm{A}^{\mathrm{n}} \Delta \mathrm{P}} \mathrm{L}_{\mathrm{m}}^{(1-\mathrm{n}) / 2} \mathrm{R}_{\mathrm{m}}(1+\mathrm{n}) / 2
\end{aligned}
$$

Onde: $\mathrm{M}$ é o índice de consistência do modelo Power-law $\left(\mathrm{Pa} . \mathrm{s}^{\mathrm{n}}\right)$, C é a concentração de sólidos na suspensão $(\% \mathrm{v} / \mathrm{v}), \Delta \mathrm{P}$ é o diferencial de pressão $(\mathrm{Pa}), \rho_{\mathrm{f}}$ é a massa específica do fluido base $\left(\mathrm{kg} \cdot \mathrm{m}^{-3}\right), \rho_{\mathrm{s}}$ é a massa específica do sólido $\left(\mathrm{kg} \cdot \mathrm{m}^{-3}\right)$, A é a área de filtração $\left(\mathrm{m}^{2}\right)$, $\left\langle\varepsilon_{\mathrm{s}}\right\rangle$ é a porosidade média da torta, $\mathrm{L}_{\mathrm{m}}$ é a espessura do meio filtrante $(\mathrm{m})$, $\mathrm{k}$ é a permeabilidade da torta $\left(\mathrm{m}^{2}\right)$ e $\mathrm{R}_{\mathrm{m}}$, a resistência do meio filtrante $\left(\mathrm{m}^{-1}\right)$.

A estimação de parâmetros foi conduzida utilizando o pacote Estima (Schwaab et al., 2007), escrito em linguagem Fortran 90, considerando mínimos quadrados ponderados como função a ser minimizada.

\section{RESULTADOS E DISCUSSÕES}

Os resultados experimentais do efeito da pressão sobre o volume de filtrado, porosidade e espessura de torta são ilustrados na Figura . Conforme citado na seção 2.1., as barras de erro experimental consideram a adoção de duas vezes o desvio padrão obtido nas triplicatas de filtração.

Observa-se que, como esperado, o aumento da pressão leva ao aumento do volume de filtrado acumulado. O diferencial de pressão é a força motriz que rege o processo de filtração. $\mathrm{O}$ aumento da pressão leva ao aumento da taxa de cisalhamento característica aplicada ao fluido, reduzindo sua viscosidade aparente e força resistiva, o que aumenta a taxa de filtração (McCABE et al., 1993).

De acordo com a Figura 3.B, não é possível identificar efeito significativo do aumento de pressão na porosidade das tortas de filtração. Por outro lado, pode-se inferir que o aumento da pressão promove o aumento da espessura da torta formada (TILLER e COOPER, 1962; CALÇADA et al., 2011). Como observado em Calçada et al. (2011) e Da Silva (2017), o aumento do fluxo de filtrado leva a maior taxa de deposição de sólidos, aumentando o número de camadas da estrutura da torta. 


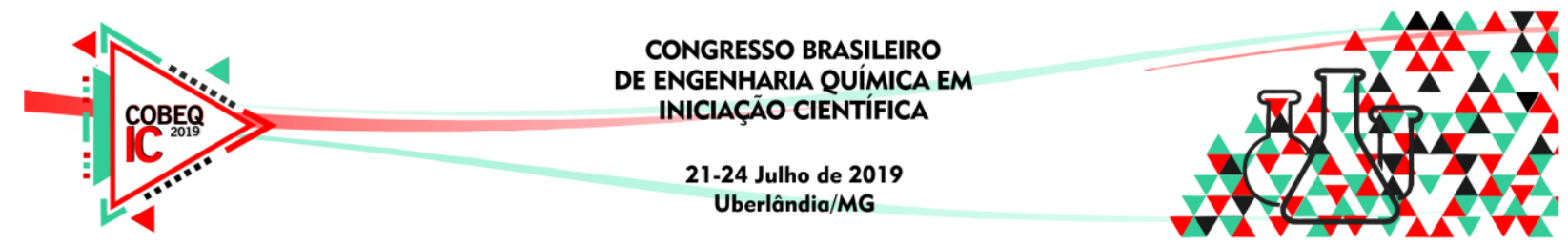

Figura 3 - Dados experimentais de filtração. Volume de filtrado acumulado (3A) e propriedades das tortas de filtração (3B).
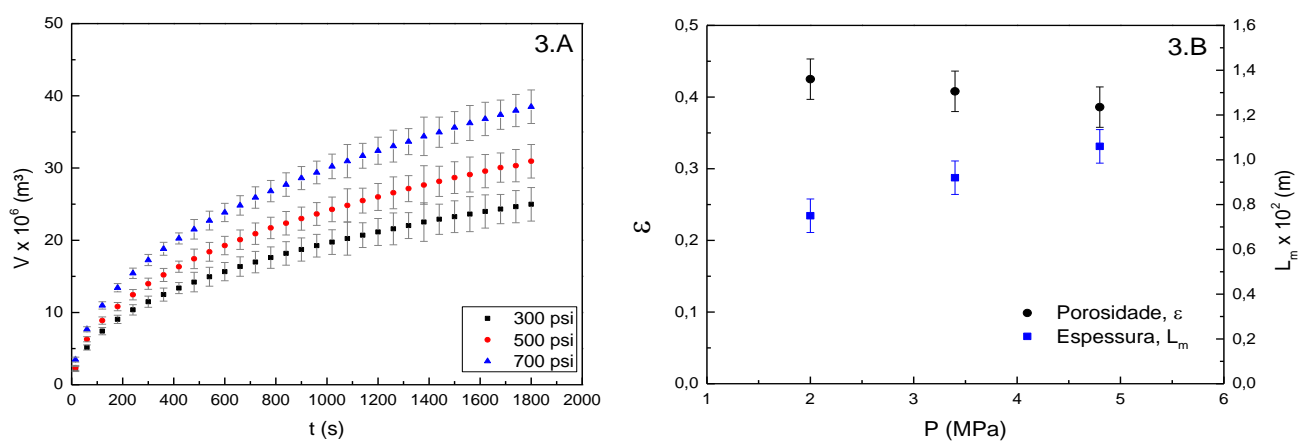

Conforme supracitado na seção 2.3., os dados de filtração foram ajustados ao modelo desenvolvido por Castro e Massarani (1981). O intervalo de confiança do modelo obtido na estimação de parâmetros é apresentado na Figura 4, assim como os parâmetros estimados para permeabilidade da torta e resistência do meio filtrante. Pode-se observar que o intervalo de confiança do modelo está de acordo com a incerteza experimental dos dados de filtração. Portanto, o modelo proposto descreve satisfatoriamente os dados de volume de filtrado.

Figura 4 - Parâmetros estimados de resistência do meio filtrante e permeabilidade da torta.
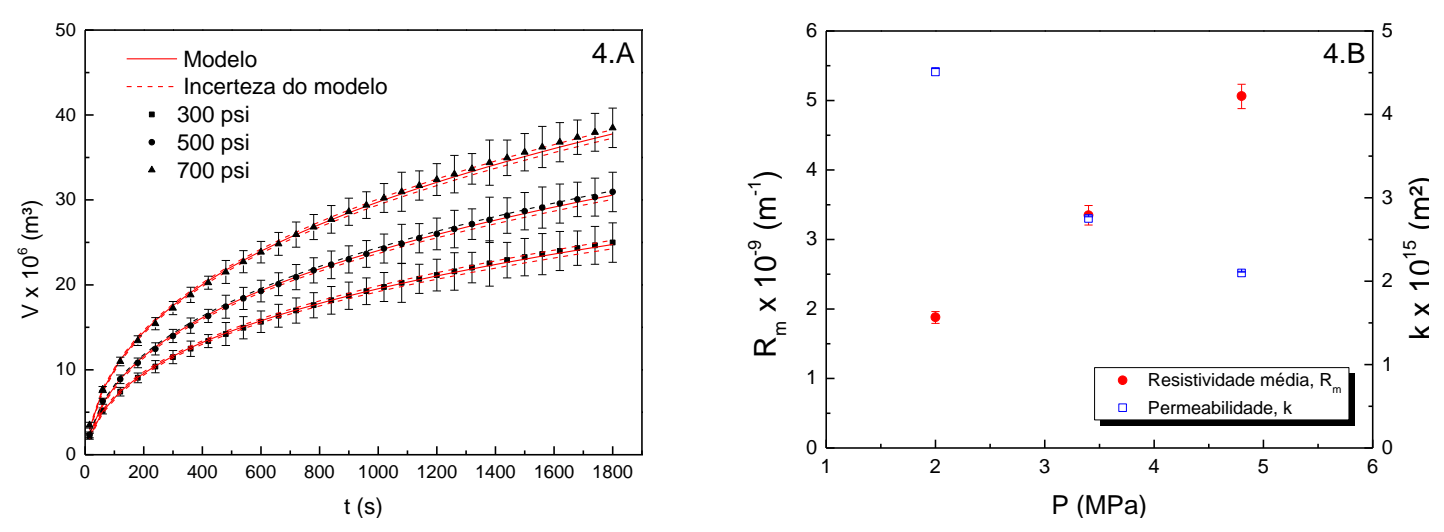

Os resultados indicam uma redução da permeabilidade das tortas de filtração com o aumento do diferencial de pressão devido aos efeitos de compressibilidade da torta. A explicação para tal fenômeno relaciona-se com a existência de variações da porosidade, uma propriedade local, possuindo valores mínimos na região próxima ao meio filtrante as partículas estão sujeitas a elevadas forças de compressão (McCABE et al., 1993; MASSARANI, 2001).

Observa-se também o crescimento da resistência do meio filtrante com o aumento da pressão aplicada. Tal fato, já esperado, é descrito por McCabe et al. (1993) onde o aumento do diferencial de pressão e a consecutiva redução da viscosidade do fluido levam ao aumento da taxa de carreamento de partículas com diâmetro inferior a faixa de diâmetros de retenção do meio filtrante. Estes parâmetros estão de acordo com aqueles obtidos em trabalhos anteriores, como em Calçada et al. (2011) e Da Silva (2017). 


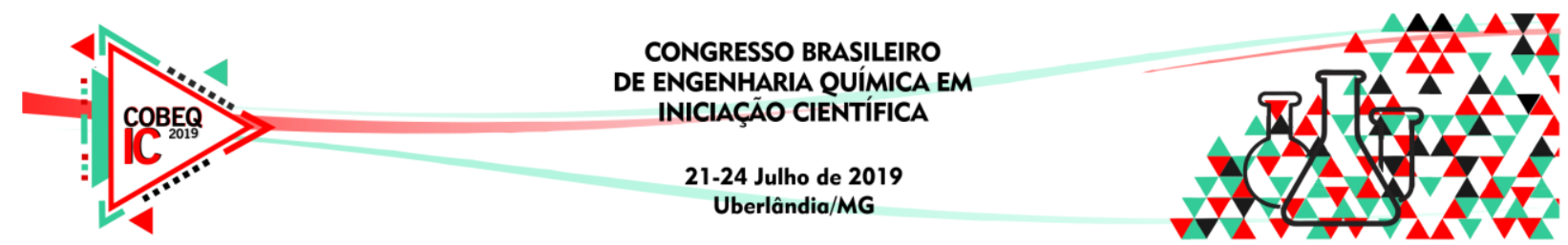

\section{CONCLUSÕES}

Resultados experimentais de filtração demonstram que há o efeito significativo da pressão no volume de filtrado acumulado. Observa-se o aumento da taxa de filtração e espessura da torta formada com o aumento do diferencial de pressão aplicado. Por outro lado, não se pode inferir que há uma diferença significativa na porosidade das tortas formadas nestas condições experimentais de pressão.

Adicionalmente, os valores estimados para permeabilidade da torta e resistência do meio filtrante demonstram-se funções do diferencial de pressão, conforme apresentado na literatura. Parâmetros estimados estão de acordo com aqueles obtidos em trabalhos anteriores. Neste cenário, a dinâmica de formação e estrutura das tortas de barita sofre ação das forças compressivas pela aplicação de um maior diferencial de pressão no processo de filtração.

\section{REFERÊNCIAS}

BÜRGUER, R., CONCHA, F., KARLSEN, K. H., Phenomenological model of filtration processes: 1. Cake formation and expression. Chemical Engineering Science, v.56, pg. 4537-4553, 2001.

DEWAN, J. T., CHENEVERT, M. E., A Model for Filtration of Water-Base Mud During Drilling: Determination of Mudcake Parameters. Journal of Petrophysics, 2001, v. 42, No3, p. 237-250.

CALÇADA, L. A.; SCHEID, C. M.; DE ARAÚJO, C. A. O.; WALDMANN, A. T. A.; MARTINS, A. L., Analysis of dynamic and static filtration and determination of mud cake parameters. Brazilian Journal of Petroleum and Gas, v.5, No3, p. 159-170, 2011.

CASTRO, L. M. N. C.; MASSARANI, G., Filtração de suspensão sólido-fluido nãoNewtoniano. Dissertação de Mestrado. Universidade Federal do Rio de Janeiro, COPPE/UFRJ/Programa de Engenharia Química, 1981.

DA SILVA, B. R. A., Avaliação da Filtração de Fluidos de Perfuração não Newtonianos Utilizando a Teoria Simplificada da Filtração. Dissertação de mestrado, Programa de Pós-Graduação em Engenharia Química, UFRRJ, Dezembro, 2017.

FERRAZ, A. S. F. S., Efeito da distribuição granulométrica de partículas sólidas e de polímeros aniônicos na formação da torta de filtração e no volume de filtrado. Dissertação de mestrado, Programa de Pós-Graduação em Engenharia Química, UFRRJ, Agosto, 2014.

MASSARANI, G., Fluidodinâmica em sistemas particulados. Programa de Engenharia Química, COPPE/Universidade Federal do Rio de Janeiro, Ed.2, 2001.

MCCABE, W. L.; SMITH, J. C.; HARRIOT, P., Unit operations of Chemical Engineering, fifth edition, McGraw-Hill, U.S., 1993.

NYGAARD, G., NÆVDAL, G., Nonlinear model predictive control scheme for stabilizing annulus pressure during oil well drilling, Journal of Process Control, v.16, p. 719-732, 2006.

RUTH, R. F., Studies in filtration, Industrial and Engineering Chemistry, p. 708-723, Junho, 1935.

SCHWAAB, M.; BISCAIA, E. C.; MONTEIRO JR, J. L.; PINTO, J. C, Nonlinear parameter estimation through particle swarm optimization, Chemical Engineering Science, v.63, p. 1542-1552, 2007. 